\title{
Blackbox Macro-modeling of the Nonlinearity Based on Volterra Series Representation of X-Parameters
}

\author{
Xiaoyan Y.Z. Xiong and Li Jun Jiang \\ Department of Electrical and Electronic Engineering \\ The University of Hong Kong \\ Pokfulam Road, Hong Kong \\ xyxiong@eee.hku.hk, jianglj@hku.hk
}

\author{
José E. Schutt-Ainé and Weng Cho Chew \\ Department of Electrical and Computer Engineering \\ University of Illinois at Urbana-Champaign \\ Urbana, IL 61801, USA \\ jesa@illinois.edu,w-chew@uiuc.edu
}

\begin{abstract}
Volterra series representation is a powerful mathematical model for nonlinear devices. However, the difficulties in determining higher-order Volterra kernels limited its broader applications. This paper proposed a systematic approach that enables a convenient extraction of Volterra kernels from $\mathrm{X}$ parameters for the first time. Then the Vandermonde method is employed to separate different orders of Volterra kernels at the same frequency, which leads to a highly efficient extraction process. The proposed Volterra series representation based on $\mathrm{X}$ parameters is further benchmarked for verification. The proposed new algorithm is very useful for the blackbox macro-modeling of nonlinear devices and systems.
\end{abstract}

Keywords-Volterra series, X-parameters, macro-modeling.

\section{INTRODUCTION}

The macro-modeling of nonlinear devices and systems is a topic of growing interest. However, it is difficult to find accurate and efficient models to characterize the nonlinear behavior of devices under arbitrary loads and input signals. X-parameters are a superset of S-parameters and have been successfully used to describe the behavior of nonlinear devices [1]-[3]. They are used to describe the numerical relationships of frequency components between different ports under certain large signal operating points (LSOPs). In this descriptive function concept, input signals are represented by a fundamental component consisting of LSOPs superposed with small harmonics. Consequently, X-parameter models are limited for transient simulation situations and they have difficulty in handling input signals with high peak-to-average ratios that will excite the device over the full linear and nonlinear operating ranges.

The Volterra series representation is another popular blackbox behavioral model of nonlinear devices with memory [4]. It can support transient simulation and is valid for signals that can excite both linear and nonlinear responses. The difficuly in determining higher-order Volterra kernels (VKs) restricted its application to only weakly nonlinear devices and systems. The extraction of Volterra kernels from X-parameters was mentioned in [5]. The novel contribution of this paper is that for the first time a systematic method for the efficient extraction of Volterra kernels from X-parameters is presented. The generalized relationship between Volterra kernels and Xparameters is explicitly formulated. In addition, it provides a complete description of Volterra kernels. Using the Vandermonde method, the kernels contributing to the same output frequency component are further separated, which leads to a very efficient process for the determination of Volterra kernels.

The organization of the paper is as follows. Section II provides a brief description of Volterra series theory. Section III gives the $\mathrm{X}$-parameter formalism with incommensurate multi-tone input. Section IV presents the detailed technical description of the Volterra kernel extraction process. In section $\mathrm{V}$, numerical example of extracting Volterra series from the $\mathrm{X}$-parameters is provided to validate the proposed method. Finally, a conclusion is given in Section VI.

\section{Volterra Series}

\section{A. Volterra Series Representation}

Volterra series are useful for describing the input/output relationship of nonlinear systems with memory. Suppose the input signal is $u(t)$, the output $y(t)$ can be represented as

$$
y(t)=\sum_{n=1}^{\infty} y_{n}(t)
$$

with

$$
\begin{aligned}
y_{n}(t)= & \frac{1}{n !} \int_{-\infty}^{+\infty} h_{n}\left(t_{1}, t_{2}, \ldots, t_{n}\right) u\left(t-t_{1}\right) \\
& u\left(t-t_{2}\right) \ldots u\left(t-t_{n}\right) d t_{1} d t_{2} \ldots d t_{n}
\end{aligned}
$$

where $h_{n}\left(t_{1}, t_{2}, \ldots, t_{n}\right)$ is called the $n$-th Volterra kernel in the time domain [4].

\section{B. Determination of the Kernels}

The harmonic input method is one of the commonly used methods for determining the Volterra kernels [4]. In general, to get the $M$-th order Volterra kernel, a $M$-tone excitation is required. Suppose the input signal is the superposition of $M$ incommensurate tones:

$$
u(t)=\sum_{m=1}^{M} \frac{V_{m}}{2} e^{j \omega_{m} t}+\text { c.c. }=\sum_{\substack{m=-M \\ m \neq 0}}^{M} \frac{V_{m}}{2} e^{j \omega_{m} t}
$$

where "c.c." denotes the complex conjugate terms, $\omega_{-m}=$ $-\omega_{m}$, and $V_{-m}=V_{m}^{*}$ for $m>0$. Here, $V_{m}$ is dimensionless and $m$ is an integer.

The $n$-th order of the output is represented in (4) with $m_{i} \neq 0$ and $d \bar{t}=d t_{1} d t_{2} \ldots d t_{n} . H_{n}\left(\omega_{m_{1}}, \omega_{m_{2}}, \ldots, \omega_{m_{n}}\right)$ 


$$
\begin{aligned}
& y_{n}(t)=\frac{1}{n !} \int_{-\infty}^{+\infty} h_{n}\left(t_{1}, t_{2}, \ldots, t_{n}\right) \cdot \prod_{i=1}^{n}\left[\frac{V_{1}}{2} e^{j \omega_{1}\left(t-t_{i}\right)}+\frac{V_{2}}{2} e^{j \omega_{2}\left(t-t_{i}\right)}+\cdots+\frac{V_{M}}{2} e^{j \omega_{M}\left(t-t_{i}\right)}+\text { c.c. }\right] d \bar{t} \\
& =\frac{1}{n !} \sum_{m_{1}=-M}^{M} \sum_{m_{2}=-M}^{M} \ldots \sum_{m_{n}=-M}^{M}\left[\prod_{i=1}^{n} \frac{V_{m_{i}}}{2}\right] H_{n}\left(\omega_{m_{1}}, \omega_{m_{2}}, \ldots, \omega_{m_{n}}\right) \cdot \exp \left(j \sum_{i=1}^{n} \omega_{m_{i}} t\right) \\
& G_{\left[k_{1}+r_{1}, r_{1}\right],\left[k_{2}+r_{2}, r_{2}\right], \ldots,\left[k_{M}+r_{M}, r_{M}\right]}\left(\omega_{1}, \omega_{2}, \ldots, \omega_{M}\right)=\frac{n !}{\left(k_{1}+r_{1}\right) ! r_{1} !\left(k_{2}+r_{2}\right) ! r_{2} ! \ldots\left(k_{M}+r_{M}\right) ! r_{M} !} \\
& \cdot H_{n}(\underbrace{\omega_{1}, \omega_{1}, \ldots, \omega_{1}}_{k_{1}+r_{1}} ; \underbrace{-\omega_{1},-\omega_{1}, \ldots,-\omega_{1}}_{r_{1}} ; \ldots ; \underbrace{\omega_{M}, \omega_{M}, \ldots, \omega_{M}}_{k_{M}+r_{M}} ; \underbrace{-\omega_{M},-\omega_{M}, \ldots,-\omega_{M}}_{r_{M}}) \\
& y(t)=\sum_{n=1}^{\infty} y_{n}(t)=\sum_{r_{1}=0}^{\infty} \sum_{r_{2}=0}^{\infty} \cdots \sum_{r_{M}=0}^{\infty}\left[\frac{\left(V_{1} / 2\right)^{k_{1}+r_{1}}\left(V_{1}^{*} / 2\right)^{r_{1}} \ldots\left(V_{M} / 2\right)^{k_{M}+r_{M}}\left(V_{M}^{*} / 2\right)^{r_{M}}}{\left(k_{1}+r_{1}\right) ! r_{1} !\left(k_{2}+r_{2}\right) ! r_{2} ! \ldots\left(k_{M}+r_{M}\right) ! r_{M} !}\right] \\
& \cdot G_{\left[k_{1}+r_{1}, r_{1}\right],\left[k_{2}+r_{2}, r_{2}\right], \ldots,\left[k_{M}+r_{M}, r_{M}\right]}\left(\omega_{1}, \omega_{2}, \ldots, \omega_{M}\right) \cdot \exp \left[j\left(k_{1} \omega_{1}+k_{2} \omega_{2}+\cdots+k_{M} \omega_{M}\right) t\right]
\end{aligned}
$$

is the $n$-th symmetric frequency domain Volterra kernel or transfer function. Many terms in (4) contain identical exponents. By collecting all those terms at frequency $\left(k_{1} \omega_{1}+k_{2} \omega_{2}+\cdots+k_{M} \omega_{M}\right) \quad\left(k_{m}\right.$ are integers and $0 \leq$ $\left.k_{m} \leq n\right)$, we could get a simplified Volterra kernel representation $G_{n}$. The relationship between $G_{n}$ and $H_{n}$ is written in (5) with $k_{1}+2 r_{1}+k_{2}+2 r_{2}+\cdots+k_{M}+2 r_{M}=n$, $r_{m}$ are integers and $r_{m} \geq 0$. The first $k_{m}+r_{m}$ arguments of $H_{n}$ are $+\omega_{m}$, the next $r_{m}$ terms are $-\omega_{m}$. For $k_{m}<0$, the signs of $\omega_{m}$ are reversed. The general representation for all the frequency components of the output signal due to an $M$-tone excitation is given in (6). The Volterra kernels can be determined by measuring the magnitudes and phases of the corresponding frequency components of the output signal.

\section{X-PARAMETER FORMALISM}

$\mathrm{X}$-parameters are the extension of S-parameters and can be used to describe the behavior of nonlinear devices in the frequency domain. For better clarification and without losing generality, we take the incommensurate three-tone excitation case to illustrate the formalism of the X-parameters [6]. Suppose the incident signal $A_{q}(t)$ at port $q$ has three large fundamental incommensurate tones, the scattered signal at port $p$ contains numerous frequency components. They are the combinations of the input tones as $\omega=n \omega_{1}+m \omega_{2}+l \omega_{3}$ and can be indexed as $B_{p,[n, m, l]}$. Then, X-parameters are used to link the scattered signal $B$ with incident signal $A$ by

$$
\begin{aligned}
& B_{p,[n, m, l]}=X_{p,[n, m, l]}^{(F)} P_{[1,0,0]}^{n} P_{[0,1,0]}^{m} P_{[0,0,1]}^{l} \\
+ & \sum_{q, n^{\prime}, m^{\prime}, l^{\prime}} X_{p,[n, m, l] ; q,\left[n^{\prime}, m^{\prime}, l^{\prime}\right]}^{(S)} P_{[1,0,0]}^{n-n^{\prime}} P_{[0,1,0]}^{m-m^{\prime}} P_{[0,0,1]}^{l-l^{\prime}} A_{q,\left[n^{\prime}, m^{\prime}, l^{\prime}\right]} \\
+ & \sum_{q, n^{\prime}, m^{\prime}, l^{\prime}} X_{p,[n, m, l] ; q,\left[n^{\prime}, m^{\prime}, l^{\prime}\right]}^{(T)} P_{[1,0,0]}^{n+n^{\prime}} P_{[0,1,0]}^{m+m^{\prime}} P_{[0,0,1]}^{l+l^{\prime}} A_{q,\left[n^{\prime}, m^{\prime}, l^{\prime}\right]}^{*}
\end{aligned}
$$

The sum runs over all $q$ and all integers $n^{\prime}, m^{\prime}, l^{\prime}$. The $\mathrm{X}$ parameters are determined under specific LSOPs (with certain DC bias and the magnitudes of the input tones). The $X^{(F)}$ term includes the information of the large signal input tones. $P_{[1,0,0]}, P_{[0,1,0]}, P_{[0,0,1]}$ are the initial phases of each tone.

\section{VOLTERRA KERNEL EXTRACTION FROM X-PARAMETERS}

\section{A. Relationship between Volterra Kernels and X-parameters}

The determination of the $M$-th Volterra kernel requires a $M$-tones input signal. For better presentation, the $M=3$ case is used to illustrate the relationship between Volterra kernels and X-parameters. The maximum mixing order is set to $M_{0}=$ $M$. In addition, thanks to the time invariant property, the initial phase of each tone is set to zero $\left(V_{m}=V_{m}^{*}\right)$. Based on (6) and (7), we could link the Volterra kernels and X-parameters by equating the same frequency component of the output signal, that is, setting $Y_{[n, m, l]}\left(n \omega_{1}+m \omega_{2}+l \omega_{3}\right)=B_{p,[n, m, l]}$. Take the output frequency with index $[n, m, l]=[0,0,1]$ as an example, the output frequency is $\omega=n \omega_{1}+m \omega_{2}+l \omega_{3}=\omega_{3}$. According to (6), the phasor of this frequency component can be written as:

$$
\begin{aligned}
Y_{[0,0,1]}\left(\omega_{3}\right)= & \sum_{r=0}^{\infty}\left[\frac{\left(V_{1} / 2\right)^{2 r_{1}}\left(V_{2} / 2\right)^{2 r_{2}}\left(V_{3} / 2\right)^{1+2 r_{3}}}{\left(r_{1} !\right)^{2}\left(r_{2} !\right)^{2}\left(1+r_{3}\right) ! r_{3} !}\right](8) \\
& \cdot G_{\left[0+r_{1}, r_{1}\right],\left[0+r_{2}, r_{2}\right],\left[1+r_{3}, r_{3}\right]}\left(\omega_{1}, \omega_{2}, \omega_{3}\right) \\
= & \frac{V_{3}}{2} G_{1}\left(\omega_{3}\right)+\frac{V_{3}^{3}}{16} G_{3}\left(\omega_{3}, \omega_{3},-\omega_{3}\right)+ \\
& \frac{V_{3} V_{2}^{2}}{8} G_{3}\left(\omega_{3}, \omega_{2},-\omega_{2}\right)+ \\
& \frac{V_{3} V_{1}^{2}}{8} G_{3}\left(\omega_{3}, \omega_{1},-\omega_{1}\right)+ \\
& \frac{V_{3}^{5}}{384} G_{5}\left(\omega_{3}, \omega_{3}, \omega_{3},-\omega_{3},-\omega_{3}\right)+\ldots \\
= & B_{p,[0,0,1]}
\end{aligned}
$$

where $\sum_{r}$ indicates a 3 -fold sum of all integer indices $r_{i}$. As presented in (8), the output at frequency $\omega_{3}$ contains different order of Volterra kernels: the linear term $G_{1}\left(\omega_{3}\right)$, the compression term $G_{3}\left(\omega_{3}, \omega_{3},-\omega_{3}\right) \triangleq G_{3 c}$, the desensitization 
terms $G_{3}\left(\omega_{3}, \omega_{2},-\omega_{2}\right) \triangleq G_{3 d 2}, G_{3}\left(\omega_{3}, \omega_{1},-\omega_{1}\right) \triangleq G_{3 d 1}$ and other higher-order terms $(n>3)$. We need to separate the different order of kernels.

\section{B. The Separation of Volterra Kernels}

In general, the magnitude of high-order output decreases drastically as the magnitude of the input signal decreases even though the magnitude of higher-order kernel may be larger. The separation of Volterra kernels depends on this fact. Provided that the magnitude of the input signal $u(t)$ is smaller than some upper bound, the high order terms above $M$ are negligible. By setting an input signal with suitable magnitude, the infinite summation for each frequency component is truncated to a finite one by ignoring the higher-order terms. Then the kernels can be separated based on the Vandermonde method [7] in the frequency domain. The basic idea consists of arranging the magnitudes of the input tones so that a matrix similar to the Vandermonde matrix can be constructed for the kernels. Take (8) as example and ignore the higher-orders with $n>3$, by changing $V_{3}$, a matrix equation is constructed:

$$
\begin{aligned}
& {\left[\begin{array}{llll}
\frac{V_{3}^{(1)}}{2} & \frac{\left(V_{3}^{(1)}\right)^{3}}{16} & \frac{V_{3}^{(1)} V_{2}^{2}}{8} & \frac{V_{3}^{(1)} V_{1}^{2}}{8} \\
\frac{V_{3}^{(2)}}{2} & \frac{\left(V_{3}^{(2)}\right)^{3}}{16} & \frac{V_{3}^{(2)} V_{2}^{2}}{8} & \frac{V_{3}^{(2)} V_{1}^{2}}{8} \\
\frac{V_{3}^{(3)}}{2} & \frac{\left(V_{3}^{(3)}\right)^{3}}{16} & \frac{V_{3}^{(3)} V_{2}^{2}}{8} & \frac{V_{3}^{(3)} V_{1}^{2}}{8} \\
\frac{V_{3}^{(4)}}{2} & \frac{\left(V_{3}^{(4)}\right)^{3}}{16} & \frac{V_{3}^{(4)} V_{2}^{2}}{8} & \frac{V_{3}^{(4)} V_{1}^{2}}{8}
\end{array}\right]\left[\begin{array}{c}
G_{1} \\
G_{3 c} \\
G_{3 d 2} \\
G_{3 d 1}
\end{array}\right] } \\
= & {\left[\begin{array}{llll}
Y\left(\omega_{3}\right)^{(1)} & Y\left(\omega_{3}\right)^{(2)} & Y\left(\omega_{3}\right)^{(3)} & Y\left(\omega_{3}\right)^{(4)}
\end{array}\right]^{T} }
\end{aligned}
$$

The different orders of kernels are the solution of (9). It is essential that the magnitudes of the input tones are properly chosen. They should be smaller than some upper bound so that lower-order Volterra kernels will not be skewed by high order terms. However the magnitudes cannot be too small as well so that the higher-order terms will not be buried in the noise. One improvement is to add additional input magnitudes and use the least square solution of the resulting overdetermined equations as the estimate of the kernels.

\section{Design of the Input Signal}

To obtain the complete description of the Volterra kenels $G_{1}\left(\omega_{1}\right), G_{2}\left(\omega_{1}, \omega_{2}\right)$ and $G_{3}\left(\omega_{1}, \omega_{2}, \omega_{3}\right)$, frequency sweep of $\omega_{1}, \omega_{2}$ and $\omega_{3}$ in the interested region is required. In addition, for a convenient kernel separation, the frequencies of the output shall be distinct from each other. Hence, careful attention must be paid in choosing the frequency components included in the input signal so that the output frequencies will not overlap with each other. Suppose the input frequencies are chosen as $\left(\omega_{1}, \omega_{2}, \omega_{3}\right)=(P, Q, R) \omega_{0}$. It is important to choose $(P, Q, R)$ carefully so that the output frequencies $\omega=(n P+$ $m Q+l R) \omega_{0}$ will not overlap with each other. Moreover, since the Volterra kernels have both permutation symmetry property (e.g., $\left.G_{3}\left(\omega_{1}, \omega_{2}, \omega_{3}\right)=G_{3}\left(\omega_{2}, \omega_{3}, \omega_{1}\right)\right)$ and conjugate symmetry property $\left(\right.$ e.g., $\left.G_{3}\left(\omega_{1}, \omega_{2},-\omega_{3}\right)=G_{3}^{*}\left(-\omega_{1},-\omega_{2}, \omega_{3}\right)\right)$, for each triplet $(P, Q, R)$, as shown in Fig. 1, we will have $6 \times 6 \times 6=216$ points in the $G_{3}\left(\omega_{1}, \omega_{2}, \omega_{3}\right)$ space (only 28 points need to be determined due to the symmetry properties). Therefore we only need to sweep $\omega_{0}$ on one frequency axis, e.g., the $\omega_{1}$ axis. The other two axes will have the same frequency sampling distribution. This leads to a very efficient frequency sweep scheme. As a comparison, in the techniques presented in literatures, the determination of $G_{3}\left(\omega_{1}, \omega_{2}, \omega_{3}\right)$ required $N^{3}$ calculation points (assuming that each frequency axis has $N$ frequency sampling points), while in this paper, by completely separating the Volterra kernels, only $N / 3$ frequency sampling points are required. Therefore, the extraction process is efficient.

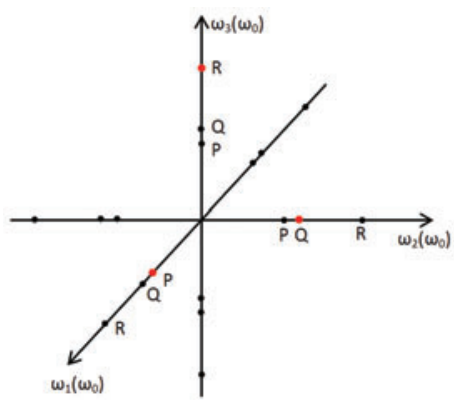

Fig. 1. Distribution of points in the $G_{3}\left(\omega_{1}, \omega_{2}, \omega_{3}\right)$ space with one triplet $(P, Q, R) \omega_{0}$.

\section{NumericAl EXAMPLE}

The Volterra series representation of a power amplifier is extracted from the X-parameters. The inset in Fig. 2(a) shows the macro-model of the amplifier. The 3-tone X-parameters of the amplifier are generated by applying the ADS X-parameter generator [8]. The frequency sweep region is $100 \mathrm{~Hz}-2 \mathrm{GHz}$ with 30 frequency sampling points. To separate different order of Volterra kernels, the power of each input tone is set to $P_{\text {in }}=[-40,-20,-15,-10,-5] \mathrm{dBm}$. The proposed method described in Sec. IV is used to extract the Volterra kernels $G_{1}\left(f_{1}\right), G_{2}\left(f_{1}, f_{2}\right)$ and $G_{3}\left(f_{1}, f_{2}, f_{3}\right)$. Fig. 2 shows the magnitudes of the Volterra kernels. As shown in Fig. 2(a), the linear transfer function $G_{1}(f)$ is identical to the S-parameters of the amplifier with small signal input. Because of the permutation and conjugate symmetry property, the magnitude distribution of $G_{2}\left(f_{1}, f_{2}\right)$ in Fig. 2(b) has two symmetry planes: $f_{1}=f_{2}$ and $f_{1}=-f_{2}$. Fig. 2(c) presents a slice of the third order Volterra kernel $G_{3}\left(f_{1}, f_{2}, f_{3}=f_{c}\right)$ with $f_{c}=1 \mathrm{GHz}$. It has a symmetry plane with $f_{1}=f_{2}$ due to the permutation symmetry property.

To validate the accuracy of the Volterra kernel separation and extraction process, Fig. 3 shows the output power of the fundamental frequency components with the increasing power of a single tone input at $1 \mathrm{GHz}$. The dashed line is obtained from the Volterra series representation with the highest order $M=7$. The dash-dotted line is simulated by the ADS harmonic balance (HB) simulator with the circuit model. Fig. 4 presents the time domain output signal calculated by the Volterra series representation and the ADS HB simulator with the input power equal to $-5 \mathrm{dBm}$. In both figures, the results obtained from the Volterra series representation agree well with those simulated by the ADS HB simulator based on the circuit model, which verifies the capability of the Volterra series for characterizing the behavior of nonlinear devices.

\section{CONCLUSION}

This paper presents a novel systematic method for extracting Volterra series representation from X-parameters. By 


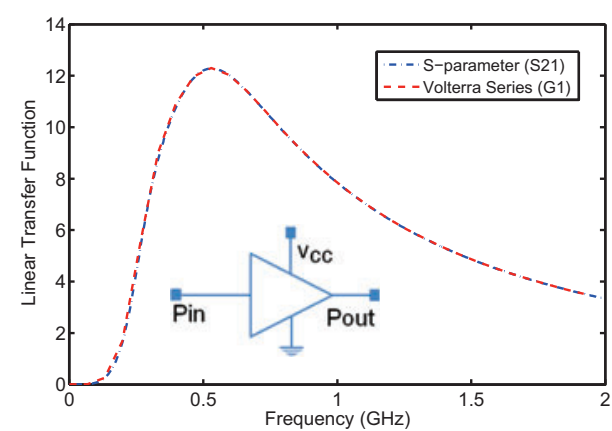

(a)

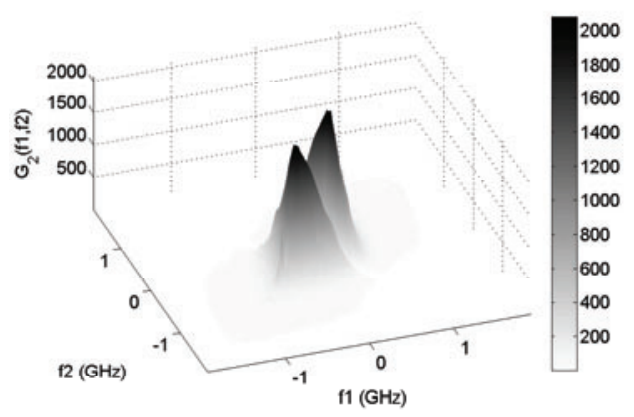

(b)



(c)

Fig. 2. Magnitudes distribution of Volterra kernels of the amplifier extracted from 3-tone X-parameters. (a) the linear transfer function $G_{1}(f)$; the inset shows the macro-model of the amplifier. (b) the second order Volterra kernel $G_{2}\left(f_{1}, f_{2}\right)$; (c) the slice of the third order volterra kernel $G_{3}\left(f_{1}, f_{2}, f_{3}\right)$ with $f_{3}$ fixed to $1 \mathrm{GHz}$.

completely separating different order of Volterra kernels based on the Vandermonde method, the complete description of Volterra kernels can be determined very efficiently. Numerical results show the capability of Volterra series representation for describing nonlinear devices in a broad input power region. The procedure for extracting Volterra kernels with the truncation order $M=3$ is illustrated in detail. The paper gives the general relationship between Volterra series and X-parameters and the method can be applied to the extraction of higher-order Volterra kernels.

\section{ACKNOWLEDGMENT}

This work was supported in part by NSFC 61271158, US AOARD 124082 contracted through UTAR, Hong Kong UGC AoE/P-04/08, and by the US National Science Foundation Award 1218552. The authors thank Agilent Technologies Inc.,

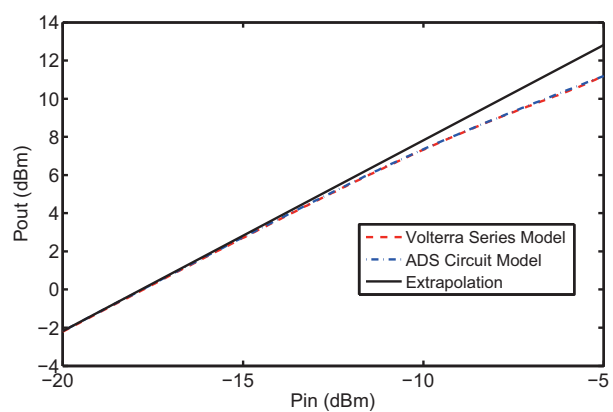

Fig. 3. The input/output power relationship of the fundamental frequency component simulated by the Volterra series model and the circuit model with a single tone input signal with frequency $f=1 \mathrm{GHz}$.

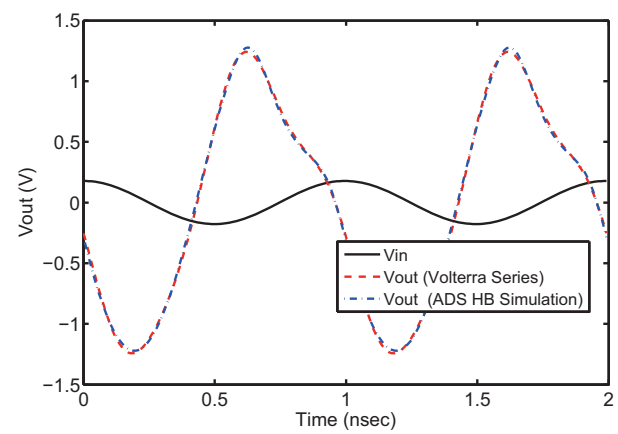

Fig. 4. The time domain representation of the output signal calculated based on the Volterra series model and the ADS harmonic balance (HB) simulator with circuit model. The input signal is a single tone with frequency $f=1 \mathrm{GHz}$ and power $P_{i n}=-5 \mathrm{dBm}$.

for supporting this work and providing the ADS X-parameter generation platform, especially David Root, Loren Betts, Steve Fulwider and Bill Wallace for fruitful discussions, insightful comments and helpful suggestions. X-parameters is a registered trademark of Agilent Technologies.

\section{REFERENCES}

[1] D. E. Root, J. Verspecht, D. Sharrit and J. Wood, "Broad-band polyharmonic distortion behavioral models from fast automated simulations and large signal vectorial network measurements," IEEE Trans. Microw. Theory Tech. pp.3656-3664, 2005.

[2] T. M. Comberiate and J. E. Schutt-Aine, "Modeling I/O Buffers Using X-Parameters," in 2013 IEEE 22th Conference on Electrical Performance of Electronic Packaging and Systems, EPEPS 2013, pp. 25-28, 2013.

[3] N. Huang and L.J. Jiang, "Simulations of pulse signals with Xparameters," IEEE Conference on Electrical Performance of Electronic Packaging and Systems, EPEPS 2011, pp. 23-26, 2011.

[4] W. J. Rugh, Nonlinear System Theory: The Volterra/Wiener Approach, The Johns Hopkins University Press, 1981.

[5] L. Sang, J. Wang, Y. Xu and R. Xu, "Extracting Volterra series representation from X-parameters for the modeling of microwave device" Journal of Electromagnetic Waves and Applications, vol. 27, pp.299308, 2013.

[6] D. E. Root, J. Verspecht, J. Horn and M. Marcu, X-Parameters: Characterization, Modeling, and Design of Nonlinear RF and Microwave Components Cambridge University Press, Sep, 2013.

[7] S. Boyd, Y. S. Tang and L. O. Chua, "Measuring Volterra kernels", IEEE Trans. Circuits and Systems, CAS-30, pp.571-577, 1983.

[8] Agilent Advanced Design System, Version 2011.10. Copyright (c) 19832011, Agilent Technologies. 